\title{
AVISO
}

Por no disponer de los correspondientes originales informáticos, la maquetación de este artículo difiere de la del publicado en papel. Por lo demás, los contenidos no han sufrido ninguna alteración.

Artículo publicado en el fascículo $2^{\circ}$ del tomo LXV (1997) de EMERITA, pp. 189-193

Autor: N. N. Kazansky

\section{LES NOMS MYCÉNIENS DANS LES TEXTES HITTITES}

E. Forrer's interpretation of Hittite ${ }^{m}$ Tawagalawa as a compounded Greek PN has been widely accepted. His equation ${ }^{m}$ Tawagalawa $={ }^{\prime} E \tau \varepsilon F_{0}{ }_{0} \lambda \varepsilon_{\varepsilon} F_{\varepsilon \zeta}$, however, causes problems due to the presence of of initial syllable 'E- in Greek, $a$ - timbre of vowel, and the sequence -ga-la- instead of -ak-la- in Hittite. It seems more plausible to segment the name as T(a)wako-lâwos which finds a perfect parallel in Greek PN $\Sigma \alpha \kappa o ́ \lambda \alpha o \varsigma$, 'The shield of the people'.

En 1924 parut l'article de E. Forrer sur les noms des Grecs dans les textes hittites ${ }^{1}$. Cet article a donné issue à des multiples études et commentaires ${ }^{2}$. À présent on peut dire que seulement deux des interprétations de E. Forrer sont

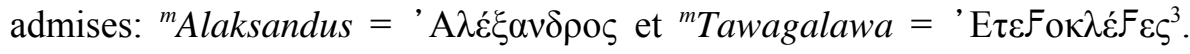
Mais aujourd'hui il faudrait réexaminer le problème en prenant en considération les données mycéniennes.

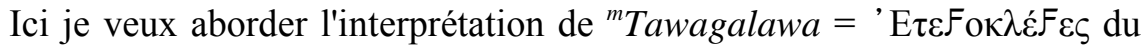
point de vue mycénien. L'interprétation de E. Forrer pose au moins trois questions qui restent sans réponse:

- Pourquoi la forme hittite a-t-elle perdu la première syllabe bien que l'écriture cunéiforme hittite eût les moyens de la noter?

- Pourquoi les scribes hittites font-ils usage du vocalisme /a/, tandis que dans la forme grecque ' $\mathrm{\varepsilon} \varepsilon F_{0} \circ \lambda \varepsilon \dot{F} \varepsilon \varsigma$ domine le timbre /e/?

- Pourquoi dans la transcription hittite on ne trouve pas de signe pour noter la syllabe fermée (le signe - $a k-$ )?

1 E. Forrer, «Vorhomerische Griechen in den Keilschrifttexten von Bogazköi», MDOG 63, 1924, pp. 1-22; E. Forrer, «Die Griechen in den Bogazköi-Texten», OLZ 27, 1924, pp. 113-118.

2 Le plus vaste en était celui de F. Sommer, «Die Ahhijavâ-Urkunden», Abhand. Bayer. Akad. Wiss., phil.-hist. Abt., NF 6, 1932, pp. 1-469.

3 E. Laroche, Les noms des hittites, Paris, 1966, p. 317. 
Je crois que ce sont des questions de ce genre qui ont été à l'origine de l'interprétation proposée par O. R. Gurney ${ }^{4}$ : ${ }^{m}$ Tawagalawa $=\Delta \varepsilon v \kappa \alpha \lambda i \omega v$. Elle est bien sûr à rejeter, car les correspondances phonétiques ne sont pas crédibles, vu la forme mycénienne de-u-ka-ri-jo (PY An 654). Le même va pour l'interprétation par W. Willis: ${ }^{m}$ Tawagalawa $=$ Tєûкро $\varsigma$ critiquée par C. Watkins ${ }^{5}$.

Il faut reconnaître que E. Forrer a bien senti la forme composée du nom ${ }^{m}$ Tawagalawa, mais on peut douter qu'il ait bien situé la frontière

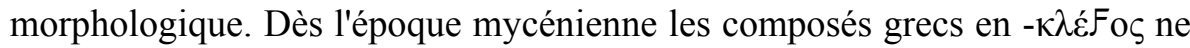
sont pas plus fréquents que ceux en $-\lambda \alpha F_{0}$ os. En mycénien on trouve e-ti-ra-

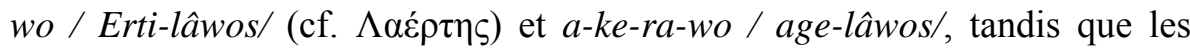
composés en - $\kappa \lambda \dot{\varepsilon} F_{0} \mathrm{\varsigma}$ apparaissent dans le patronyme e-te-wo-ke-re-wi-jo / Etewo-klewehios/.

$\mathrm{Si}{ }^{m}$ Tawagalawa est un composé en $-\lambda \bar{\alpha} F_{\mathrm{O}} \zeta$, la première partie de ce nom trouve elle aussi une interprétation évidente: T(a)wako- de *twakos, cf. skr. tvaka- 'peau', grec бókos 'bouclier'.

Le fait que dans les archives mycéniennes on ne trouve pas un mot pour 'bouclier' ne signifie pas qu'il n'ait pas existé. Dans l'épopée grecque c'est Ajax qui porte un bouclier:

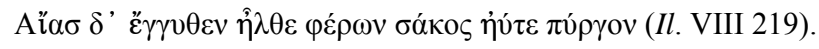

Le caractère mycénien d'Ajax était démontré ailleurs ${ }^{6}$. M. L. West a proposé une reconstruction phonétique mycénienne ${ }^{7}$ :

*Aiwans d' engúten élthe phéron twákos ewúte púrgon.

Pour le grec du II ${ }^{\text {ème }}$ mill. on a déjà supposé malgré le manque d'exemples mycéniens le groupe initial $* t w-{ }^{8}$. L'interprétation ici proposé nous en fournit

O. R. Gurney, The Hittites, 1954, p. 56.

5 Publié dans E. Vermeule, «Response to Hans Güterbock», AJA 87, 1983, p. 142 et note.

P. Cauer, Grundfragen der Homerkritik, 3.Aufl., Leipzig, 1921-1923, p. 263; D. Page, History and the Homeric Iliad, Berkeley, 1959, pp. 234-238; T. B. L. Webster, From Mycenae to Homer, London, 1958, pp. 101, 115.

7 M. L. West, «The rise of the Greek Epic», JHS 108, 1988, p. 158.

8 M. Lejeune, Phonétique historique du mycénien et du grec ancien, Paris, 1972, p.

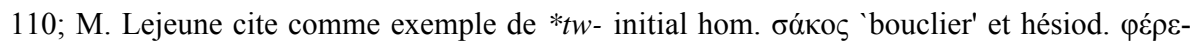
EMERITA. Revista de Lingüística y Filología Clásica (EM) - LXV 2, 1997, pp. 189-193 
un exemple.

Le nom $\Sigma \alpha \kappa o ́ \lambda \alpha F_{\text {o }}$ manque dans la tradition mythologique grecque, et cela ne doit pas nous étonner: ce personnage a exercé son activité en Lycie, loin de la Grèce et n'a pas réussi à fonder une dynastie de monarches lyciens comme le fit Mopsos en Pamphylie (hitt. cun. Muksa, myc. Mo-qo-so, en

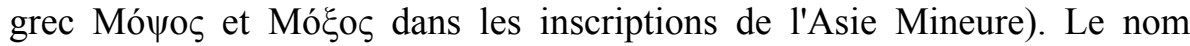
$\Sigma \alpha \kappa o ́ \lambda \alpha o \zeta$ apparait seulement dans un document grec, mais sous une forme archaïque (ou archaïsée) sans contraction: $\Sigma \alpha \kappa o ́ \lambda \alpha F_{0 \varsigma}>\Sigma \alpha \kappa o ́ \lambda \alpha \varsigma^{9}$. Il est difficile de décider si c'est la mode héllénique qui a provoqué la formation d'un nom selon les régles archaïques ${ }^{10}$ ou s'il s'agit d'un nom qui reflète une vieille tradition mythique restée inconnue.

De point de vue morphologique il faut reconnaître le caractère archaïque du composé *Twako-lâwos. Les noms en -lâwo- sont fréquents dès l'époque mycénienne. Nous en trouvons plusieurs: a-ke-ra-wo KN Vc 316; PY Cn 599, Un 1320; a-pi-ra-wo KN As 1516; ]me-ta-ra-wo KN B 799; ne-e-rawo PY Fn 79; ]e-qe-ra-wo KN B 5025; pe-ri-ra-wo PY An 654; e-ti-ra-wo $\mathrm{PY} \mathrm{CN} 131,655$. À ce groupe on rattache souvent ${ }^{11}$ le nom e-ke-ra-wo, ]eke-ri-ja-wo qui couvre peut-être /-l(l)âwôn/ > /-l(l)aun/, l'interprétation de A. Leukart, admise par Th. G. Palaima ${ }^{12}$. Dans les archives mycéniennes E-kera-wo reçoit le même lot de terre que le wanax de Pylos; l'hypotèse selon

баки́s.

9 Fr. Preisigke, Wörterbuch der griechischen Papyruskunden, III Bd., Berlin, 1931. $\Sigma \alpha \kappa o ́ \lambda \alpha F_{\text {o }}$ apparait comme un patronymique dans une longue liste des noms qui tous sont grecs malgré que les individus qui les portaient vivaient en Égypte.

10 Et en même temps analogique, car on pourrait s'attendre plutôt à la forme $* \Sigma \alpha \kappa \varepsilon \varsigma-$ $\lambda \alpha F_{\mathrm{os}}$.

11 Pour la liste des interprétations de ce nom et pour une bibliographie, voir F. Aura Jorro, Diccionario Micénico, vol. I, Madrid, 1985, pp. 210-211. L'idée de trouver dans ce nom le composé en -lâwôn ne peut pas être admise qu'avec un reserve.

12 A. Leukart, «Les signes *76 $\left(\mathrm{ra}_{2}\right.$, «rja») et *68 ( $\mathrm{ro}_{2}$ «rjo») et le nom du grand prêtre de Poseidon (sinon du roi) à Pylos», Mykenaika, J. P. Olivier (ed.), Paris, 1992 (= BCH Suppl. 25), pp. 388-390; Th. G. Palaima, «Writing in the Service of the King: Hand 24 and Special vs. Normal Mycenaean», APA Annual Meeting December 29, 1993, Washington, 1993.

EMERITA. Revista de Lingüística y Filología Clásica (EM) - LXV 2, 1997, pp. 189-193 
laquelle $E$-ke-ra-wo est le nom du roi paraît bien plausible ${ }^{13}$. L'interprétation de E-ke-ra ${ }_{2}$-wo comme /*Hekhe-l(l)âwôn/ > /*Hekhe-l(l)aun/ me paraît moins plausible ${ }^{14}$ que l'interprétation /Enkhes-lâwôn/ qui pourrait expliquer la geminée provenant du groupe $-s{ }^{15}{ }^{15}$. L'évidence linguistique est assez faible en ce qui concerne l'élément -lâwôn dans le nom /Enkhes-lâwôn/, mais la place distinguée de $E-k e-\mathrm{ra}_{2}-w o$ dans la hiérarchie mycénienne est assûrée. La place de ${ }^{m}$ Tawagalawa dans la hiérarchie sociale de l'Ahhijava paraît moins claire. Forrer voyait en ${ }^{m}$ Tawagalawa le frère du roi achéen; Sommer a très vivement attaqué cette interprétation. Il y a quelques années $\mathrm{H}$. G. Güterbock a réexaminé cette ligne du texte hittite. Dans son étude il a réhabilité la point de vue de Forrer et rétabli la parenté entre ${ }^{m}$ Tawagalawa et le roi de l'Ahhijava ${ }^{16}$.

L'ortographie mycénienne peut elle aussi renforcer mon interprétation du nom hittite. On peut noter que l'interprétation de ${ }^{m}$ Tawagalawa comme

13 Je ne veux pas dire que Enkhes-lâwôn litt. 'la lance du peuple' et Twako-lâwos litt. 'le bouclier du peuple' soient deux frères. Les dates historiques y font obstacle: Twako-lâwos a vécu avant 1255 av. J.-C., tandis que Enkhes-lâwôn était wanax au moment de la chute de Pylos (environ 1200 av. J.-C.). Tout de même il est bien probable que nous avons ici deux réprésentants d'une seule famille qui avait un penchant pour des noms composés en -lâwos. On peut noter qu'en Sparte c'était la coûtume de la famille des Egéides de porter les noms en lâwos, tandis que les Eurypontides portaient les noms en -dâmos, cf. B. Sergent, «La réprésentation spartiate de la royauté», Revue de l'histoire des religions 189, 1976.

14 Cf. Aura Jorro, Diccionario Micénico, vol. I, Madrid, 1985, pp. 210-211.

15 Comme dans la forme $a-k e-r a_{2}$-te $<>*$ agersantes.

16 «In the passage in question the words 'your brother' and Tawagalawa stand side by side, each one preceded by the same Akkadian preposition. Sommer had found no clear example of such a repetition of the preposition with a noun and its apposition. He also thought that a small trace after Tawagalawa was beginning of the Hittite enclitic -ya, 'and'. So he translated 'with my brother and Tawagalawas'. Since Sommer's time, good examples of the repeated proposition have been found in texts of Hattusilis III, and an enlarged photograph shows that the traces cannot be the beginning of -ya. Therefore I now return to Forrer's translation: 'This charioteer used to step on the chariot together with me and your brother Tawagalawas'.» (H. G. Güterbock, «The Hittites and the Aegean World: 1. The Ahhiyawa Problem Reconsidered», AJA 87, 1983, pp. 133-138, esp. p. 136).

EMERITA. Revista de Lingüística y Filología Clásica (EM) - LXV 2, 1997, pp. 189-193 
/Twako-lâwos/ pose moins de problèmes que celle de E. Forrer $\left({ }^{m}\right.$ Tawagalawa $={ }^{\prime}$ E $\left.\tau \varepsilon F_{0}{ }_{0} \lambda \varepsilon F_{0} F_{\delta}\right)$. L'interprétation phonétique devient plus précise et permet de comprendre pourquoi le scribe hittite s'est servi du timbre /a/ et de syllabes ouvertes pour transcrire ce nom en signes cunéiformes. On pourraient représenter en hittite le groupe initial *twa par ta-va ou tu(v)a-. Comme me l'a indiqué Andrei A. Koriolov (Institut de linguistique de l'Académie des Sciences de la Russie, Moscou), le deuxième moyen est plus répandu dans l'écriture hittite. L'ortographe mycénienne connaît elle aussi ces deux moyens: ta-wa- et tu-wa-, mais en mycénien c'est le premier qui a prédominé (on a o-da-tu-we-ta / o-da-twe-ta, mais au début d'un mot da-wano KN As 1517 /Dwanos/; ta-wa-ko-to KN Od 715, qui peut contenir, malgré la morphologie douteuse la même racine *twak-). On pourrait supposer que ce n'est pas par hasard que le texte hittite comporte ${ }^{m} \mathrm{Ta}-\mathrm{va}$ pour exprimer le *twa- initial mycénien. Du moment que ${ }^{m}$ Tawagalawa a fit objet d'une correspondance entre le roi hittite et celui d'Ahhijava, on pourrait songer à l'influence directe de l'écriture mycénienne sur le texte hittite.

Le même texte hittite comporte une série de noms parmi lesquels ${ }^{m}$ Piyamaradus est sans aucun doute hittite et signifie 'Donné par le dieu Radus ${ }^{17}$, ainsi que ${ }^{m}$ Atpas qui contient le groupe phonétique -tp- qui était impossible en mycénien. On peut supposer qu'ils étaient des Anatoliens au service mycénien, comme on a à Knossos les noms pi-ja-se-me KN AS 1516 avec un élément anatolien piya- 'donner' et pi-ja-si-ro KN AS 1516 qui couvre peut-être le nom hittite Piyassili ${ }^{18}$. Mais ce n'est pas le cas de l'individu nommé ${ }^{m} L a-H U R-z i$. Il est mentionné comme un énemi qui a attaqué le roi hittite près de Iyalanda et en même temps comme un «frère du (roi de l'Ahhijava ??)». L'interprétation ingénieuse de M. L. Palmer qui lisait ${ }^{m} L a-H U R-z i$ comme $\Lambda \alpha \varepsilon ́ p \tau \eta \varsigma$ ne peut pas convaincre car elle ne repose que sur un isosyllabisme. On pourrait voir ici encore une trace de transcription de l'écriture mycénienne. En effet, c'est un peu étonnant qu'un nom étranger soit écrit avec la signe -HUR-, qui peut se lire -hur-, -har-, - mur- $^{19}$, sans compter

17 E. Laroche, Les noms des hittites, Paris, 1966, p. 317.

18 L. Baumbach, «An examination of the Personal Names in the Knossos Tablets as an Evidence for the Social Structure of Crete in the Late Minoan II Period», in: Minoan Society. Proceedings of the Cambridge Colloquium 1981, Bristol, 1983, p. 8.

19 E. Laroche, Les noms des hittites, Paris, 1966, n. 676.

EMERITA. Revista de Lingüística y Filología Clásica (EM) - LXV 2, 1997, pp. 189-193 
des lectures plus rares tels que -hir- et $-u r_{-}^{20}$. On pourrait noter que ${ }^{m} L a-$ HUR-zi peut correspondre aussi à des noms mycéniens tels que ra-u-ta (un forgeron dans PY Jn 832). Ce nom peut être égéen venant du pré-grec. Cela n'empêche pas que ${ }^{m}$ Tawagalawa soit un Mycénien portant un nom composé Twako-lâwos, ce qui a donné en grec $\Sigma \alpha \kappa o ́ \lambda \alpha o \zeta$.

N. N. KAZANSKY

20 R. Labat, Manuel d'épigraphie akkadienne, $3^{\text {ème }}$ ed., Paris, 1959, p. 187.

EMERITA. Revista de Lingüística y Filología Clásica (EM) - LXV 2, 1997, pp. 189-193 